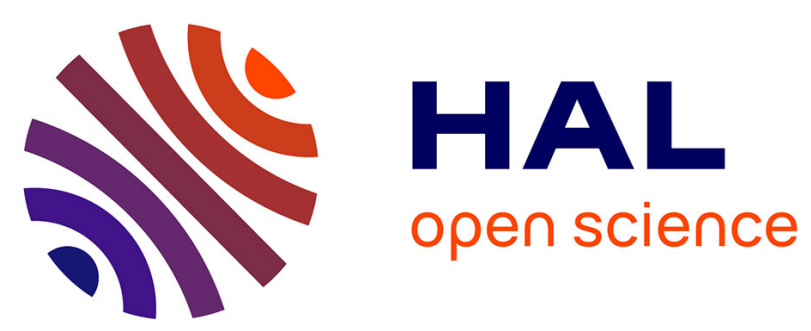

\title{
On Efficient Child Making
}

Philippe Michel, Bertrand Wigniolle

\section{To cite this version:}

Philippe Michel, Bertrand Wigniolle. On Efficient Child Making. Economic Theory, 2007, 31 (2), pp.307-326. 10.1007/s00199-006-0099-1 . halshs-00185259

\section{HAL Id: halshs-00185259 \\ https://shs.hal.science/halshs-00185259}

Submitted on 5 Nov 2007

HAL is a multi-disciplinary open access archive for the deposit and dissemination of scientific research documents, whether they are published or not. The documents may come from teaching and research institutions in France or abroad, or from public or private research centers.
L'archive ouverte pluridisciplinaire HAL, est destinée au dépôt et à la diffusion de documents scientifiques de niveau recherche, publiés ou non, émanant des établissements d'enseignement et de recherche français ou étrangers, des laboratoires publics ou privés. 


\title{
On Efficient Child Making*
}

\author{
Philippe Michel ${ }^{\dagger}$ and Bertrand Wigniolle ${ }^{\ddagger}$
}

February 23, 2006

\begin{abstract}
This paper is devoted to the study of the Pareto-efficiency of the competitive equilibrium for an overlapping generations economy with endogenous fertility. Pareto-efficiency needs a reformulation when fertility is endogenous. Then it is proved that a competitive equilibrium that converges in over-accumulation is non-Pareto-efficient. However, we provide an example in which a competitive equilibrium that converges in under-accumulation is non-Pareto-efficient. Finally, we give a general condition that ensures the Pareto-efficiency of the competitive equilibrium.
\end{abstract}

Keywords and Phrases: endogenous fertility, Pareto-efficiency.

JEL Classification Numbers: D9, J13 and D61.

${ }^{*}$ This paper was presented at the conference "Irregular Growth: Beyond Balanced Growth", Paris, June 2003. The authors would like to thank Tapan Mitra for helpful comments. They also are grateful to Claire Loupias, who began to think with them on the subject and to an anonymous referee for useful suggestions and improvements.

†GREQAM, Université de la Méditerranée and EUREQua, Université de Paris I. The current version of this paper was completed after Philippe Michel's sudden death. I want to express my deep sorrow for the loss of a close friend and an excellent economist, whom I learned a lot from over the years.

${ }^{\ddagger}$ Centre d’Economie de la Sorbonne, Université de Paris I . Address: EUREQua, Université de Paris-I, Maison des Sciences Economiques, 106-112, boulevard de l'hôpital, 75647 Paris, Cedex 13, France. Email : wignioll@univ-paris1.fr. 


\section{Introduction}

This paper is devoted to the study of the Pareto-efficiency of the competitive equilibrium for an overlapping generations economy with endogenous fertility.

The problem is well-known when fertility is exogenous. Considering the standard overlapping generations model (cf. Diamond [7]), three types of steady states may exist in a competitive economy: under-accumulation, the golden rule or over-accumulation. The two first steady states are Paretooptimal when the third is not. Moreover, for an inter-temporal dynamic equilibrium converging towards a steady state, if this steady state is in underaccumulation, the equilibrium trajectory is Pareto-optimal; if the steady state is in over-accumulation, the equilibrium trajectory is not Pareto-optimal. These results are carefully presented in De la Croix and Michel [6].

What happens to these properties when fertility is endogenous?

Before answering this question, we must clarify some basic assumptions and concepts. On the one hand, we must choose a theoretical framework that endogenizes the fertility decisions. On the other hand, we need to reconsider the notion of Pareto-efficiency in a context where fertility is endogenous: Pareto-efficiency is a notion that is basically defined for a given set of agents.

In the literature, there have been many approaches that endogenize fertility decisions. The benchmark framework consists in assuming that children are a consumption good, which appear in the utility function of the parents. The basic references are the articles by Becker [1], Willis [14] and Eckstein and Wolpin $[8]^{1}$. Moreover, we focus on the case of identical agents in each generation: we consider one representative agent by generation.

The second point at issue is the definition of the Pareto-efficiency when fertility is endogenous. We introduce a concept called "Representative-Consumer" efficiency (or RC-efficiency), which refers to an allocation (which satisfies the resource constraint of the economy), for which no other allocation exists that would lead to a higher level of utility for all generations with a strict improvement for (at least) one generation. In the sense of this definition, the only criterion is the utility of each generation. Thus, an allocation that improves the utility level of one (or many) generation(s) to the detriment of the size of one (or many) generation(s) is considered preferable. For that reason, it is possible to think of another notion, which add to the preceding notion an additional constraint: an allocation can dominate another one only if it

\footnotetext{
${ }^{1}$ Other approaches have been developed in the literature based on the additional assumption of descendant altruism, as in Becker and Barro [2]. Another way of endogenizing fertility (cf. Nishimura and Zhang [12]) is to introduce ascendant altruism and strategic behaviors of parents.
} 
includes at least an equal number of children in each period. But, as a result of our study, we will see that this second notion does not lead to a different classification of competitive equilibria. Therefore we will concentrate our study on the basic notion

We reach the following results.

First, we present the model and give the precise conditions that ensure the existence and the uniqueness of the inter-temporal equilibrium. Second, we focus on the efficiency properties of the competitive equilibrium. Clearly a competitive equilibrium that converges in over-accumulation is not RC-efficient, as a consequence of the comparison with unchanged population. More surprisingly, a competitive equilibrium that converges in underaccumulation can be RC-dominated. This result is shown by a simple example: starting from the competitive equilibrium, we build an allocation that gives a higher fertility and leaves the consumption levels unchanged.

Finally, considering a competitive equilibrium which converges in underaccumulation, we give a sufficient condition to ensure that this trajectory is RC-efficient. This condition is that the limit value of the wage does not make it possible to finance a fertility level that is higher than the limit value of the interest factor.

Our results can be compared with existing literature.

In a framework close to ours, Eckstein and Wolpin [8] focus on the optimal stationary state. They do not, however, precisely state the sufficient conditions that ensure the concavity of the optimization problem ${ }^{2}$.

As for the problem of the existence and uniqueness of the inter-temporal equilibrium with endogenous fertility, Raut and Srinivasan [13] and Chakrabarti [4] consider models that are close to ours. They particularly focus on the occurrence of multiple equilibria, indetermination and complex dynamics.

Two recent papers are concerned with the definition of Pareto-efficiency criteria in an endogenous fertility framework. Conde-Ruiz, Giménez and Perez-Nievas [5] present two definitions of Pareto efficiency that are close to ours. They apply these notions to a model in which the only productive factor is human capital, and they do not study the efficiency properties of the competitive equilibrium.

Golosov, Jones and Tertilt [9] are concerned with the definition of Paretoefficiency notions in a general framework with heterogeneous agents. They propose two notions called P-efficiency and A-efficiency. One difference with our approach is that their notion of P-efficiency depends on the utility level assigned to "unborn" agents. On the contrary, our notions of efficiency are defined independently of any reference to the utility of "unborn" agents. In

\footnotetext{
${ }^{2}$ Even if they mention that they obtain such conditions for a Cobb-Douglas economy.
} 
a framework with homogenous generations, their notion of A-efficiency corresponds to our notion of RC-efficiency. For a sufficiently low level of utility assigned to unborn agents, their notion of P-efficiency corresponds to our notion of CRC-efficiency. But the main difference with our approach is that they study a class of models with the particular notion of "P-equilibrium" which do not include our model. Indeed, for the class of models that they consider, they find that all P-equilibrium is P-efficient, when we consider a model where the equilibrium can be inefficient.

This paper is organized in the following way. Section two presents the model. Section three defines and studies the inter-temporal equilibrium. Section four defines Pareto efficiency when fertility is endogenous, and gives sufficient conditions for non-efficiency. Section five gives a sufficient condition for ensuring that a competitive equilibrium converging in under-accumulation is efficient. Section six presents our conclusions. The most demanding proofs can be found in the Appendix.

\section{The model}

We make use of an overlapping generations model. Each individual lives for two periods. She or he inelastically supplies one unit of labor during her/his youth and retires in old age. During their first period of life, agents work, consume save and choose their number of children. Children entail a cost for their parents. In their second period of life, agents consume the proceeds of theirs savings.

\subsection{Consumers}

At each date $t$, the new generation is populated with a number $N_{t}$ of identical agents. Each agent is endowed with an inter-temporal utility function:

$$
U\left(c_{t}, d_{t+1}, m_{t}\right)
$$

with $\left(c_{t}, d_{t+1}, m_{t}\right) \in \mathbb{R}_{+}^{3} \cdot c_{t}$ and $d_{t+1}$ are respectively the consumptions of good during youth and old age, and $m_{t}$ is the number of children ${ }^{3}$. The number of agents of the generation $t+1$ is thus equal to:

$$
N_{t+1}=m_{t} N_{t}
$$

\footnotetext{
${ }^{3}$ As usual, we consider $m_{t}$ as a continuous variable. This assumption is consistent with a model which assumes identical agents within each generation.
} 
Assumption $1: U$ is a function from $\mathbb{R}_{+}^{3}$ to $\mathbb{R} \cup\{-\infty\}$, and $U$ maps $\mathbb{R}_{++}^{3}$ to $\mathbb{R}$, with ${ }^{4}$ :

$$
U(\bar{c}, \bar{d}, \bar{m})=\lim _{\substack{(c, d, m) \in \mathbb{R}_{++}^{3} \\(c, d, m) \rightarrow(\bar{c}, \bar{d}, \bar{m})}} U(c, d, m) \text { for every }(\bar{c}, \bar{d}, \bar{m}) \in \mathbb{R}_{+}^{3} / \mathbb{R}_{++}^{3}
$$

$U$ is twice continuously differentiable on $\mathbb{R}_{++}^{3}$, strictly concave, increasing in each argument and satisfies the Inada conditions:

$$
\lim _{c \rightarrow 0} U_{c}^{\prime}=\lim _{d \rightarrow 0} U_{d}^{\prime}=\lim _{m \rightarrow 0} U_{m}^{\prime}=+\infty
$$

The budget constraints faced by a generation $t$ agent are:

$$
\begin{aligned}
c_{t}+\phi m_{t}+s_{t} & =w_{t} \\
d_{t+1} & =R_{t+1} s_{t}
\end{aligned}
$$

where $w_{t}$ is the wage earned during period $t, s_{t}$ the savings, and $R_{t+1}$ the gross return in $t+1$ on savings. Each child entails a constant educational cost $\phi$.

From (4) and (5), the lifetime budget constraint can be stated as:

$$
c_{t}+\frac{d_{t+1}}{R_{t+1}}+\phi m_{t}=w_{t}
$$

With assumption 1, maximizing the utility function under the lifetime budget constraint leads to the following first-order conditions, which are necessary and sufficient:

$$
U_{c t}^{\prime}=R_{t+1} U_{d_{t+1}}^{\prime}=\frac{U_{m_{t}}^{\prime}}{\phi}
$$

This enables us to write the following demand functions:

$$
\begin{aligned}
c_{t} & =c\left(w_{t}, R_{t+1}\right) \\
m_{t} & =m\left(w_{t}, R_{t+1}\right) \\
s_{t} & =s\left(w_{t}, R_{t+1}\right) \\
d_{t+1} & =d\left(w_{t}, R_{t+1}\right)
\end{aligned}
$$

Functions $c, m, s$ and $d$ are defined on $\mathbb{R}_{++}^{2}$ and are continuously differentiable.

\footnotetext{
${ }^{4}$ Equation (3) means that a unique limit exists, independent of the converging $(c, d, m)$ sequence chosen.
} 


\section{$2.2 \quad$ Firms}

At each period $t$ one competitive firm exists, which produces $Y_{t}$ quantity of good, according to a production function with two inputs (capital: $K_{t}$; labor: $L_{t}$ ), and constant returns:

$$
Y_{t}=F\left(K_{t}, L_{t}\right)
$$

The capital fully depreciates within the production period. We can define $f$ as: $f(k) \equiv F(k, 1)$.

Assumption 2: $f: \mathbb{R}_{+} \rightarrow \mathbb{R}_{+}$, and for all $k>0, f^{\prime}(k)>0$ and $f^{\prime \prime}(k)<0$.

The profit maximization of the firm gives:

$$
\begin{aligned}
& w_{t}=F_{L}^{\prime}\left(K_{t}, L_{t}\right)=f\left(K_{t} / L_{t}\right)-\left(K_{t} / L_{t}\right) f^{\prime}\left(K_{t} / L_{t}\right) \equiv w\left(K_{t} / L_{t}\right) \\
& R_{t}=F_{K}^{\prime}\left(K_{t}, L_{t}\right)=f^{\prime}\left(K_{t} / L_{t}\right) \equiv R\left(K_{t} / L_{t}\right)
\end{aligned}
$$

\section{The inter-temporal equilibrium}

\subsection{Equilibrium characterization}

The initial conditions are the following. At period $t=0, N_{-1}$ old agents and $N_{0}$ young agents are living. The old agents hold equal shares of the capital stock $K_{0}$. This stock comes from their past savings behavior: $K_{0}=N_{-1} s_{-1}$. The consumption of the old agents can then be given by:

$$
d_{0}=R_{0} K_{0} / N_{-1}
$$

Along an inter-temporal equilibrium, all markets are balanced.

The labor market is balanced at each period: $L_{t}=N_{t}$, with $N_{t}$ following the relation : $N_{t+1}=m_{t} N_{t}$. Prices result from the firms' behavior at equilibrium:

$$
R_{t}=R\left(k_{t}\right) \text { and } w_{t}=w\left(k_{t}\right) \text { with: } k_{t}=K_{t} / N_{t}
$$

The commodity market is balanced, or:

$$
F\left(K_{t}, N_{t}\right)=N_{t} c_{t}+N_{t-1} d_{t}+K_{t+1}+N_{t} \phi m_{t}
$$

Taking consumer's budget constraints into account, this last relation is equivalent to:

$$
K_{t+1}=N_{t} s_{t}
$$

Finally, using optimal behaviors and equilibrium conditions, it is possible to give the definition of the inter-temporal equilibrium: 
Definition 1 Starting from initial conditions $N_{-1}, N_{0}, K_{0}$ and $d_{0}=R_{0} K_{0} / N_{-1}$, an inter-temporal equilibrium is a sequence $\left(K_{t}, N_{t}, c_{t}, d_{t}, m_{t}\right)_{t \geq 0}$, which satisfies:

$$
\begin{aligned}
c_{t}+\frac{d_{t+1}}{R_{t+1}}+\phi m_{t} & =w_{t} \\
U_{c t}^{\prime} & =R_{t+1} U_{d_{t+1}}^{\prime}=\frac{U_{m_{t}}^{\prime}}{\phi} \\
N_{t+1} & =m_{t} N_{t} \\
F\left(K_{t}, N_{t}\right) & =N_{t} c_{t}+N_{t-1} d_{t}+K_{t+1}+N_{t} \phi m_{t} \\
\text { with } R_{t} & =R\left(k_{t}\right) \text { and } w_{t}=w\left(k_{t}\right) \text { where: } k_{t}=K_{t} / N_{t}
\end{aligned}
$$

All equilibrium variables can be expressed as functions of the variable $k_{t}$. This property leads to the following simple characterization:

Proposition 1 An inter-temporal equilibrium is characterized by a sequence $\left(k_{t}\right)_{t \geq 0}$ with $k_{t}>0$, such that: $k_{0}=K_{0} / N_{0}$ is given, and $\forall t \geq 0$,

$$
m\left[w\left(k_{t}\right), R\left(k_{t+1}\right)\right] k_{t+1}-s\left[w\left(k_{t}\right), R\left(k_{t+1}\right)\right]=0
$$

The proof is straightforward. From the sequence $\left(k_{t}\right)_{t \geq 0}$, the sequence of prices can be defined by (14). Individual variables $\left(c_{t}, s_{t}, m_{t}, d_{t+1}\right)_{t \geq 0}$ are given by (6), (7), (8) and (9). All equilibrium conditions are thus satisfied.

\subsection{Sufficient conditions for the existence and unique- ness of the inter-temporal equilibrium}

We define the function $\Delta$ as:

$$
\Delta(w, k)=m\left(w, f^{\prime}(k)\right) k-s\left(w, f^{\prime}(k)\right)
$$

$\Delta$ is defined on $\mathbb{R}_{++}^{2}$ and is continuously differentiable. The dynamic equation (15) can be written as:

$$
\Delta\left(w\left(k_{t}\right), k_{t+1}\right)=0
$$

A sufficient condition for the existence of an inter-temporal equilibrium $\left(k_{t}\right)_{t \geq 0}$ is that for all $w>0$, there exists $k>0$, such that $\Delta(w, k)=0$. Moreover, if $\forall w>0$, the equation $\Delta(w, k)=0$ has a unique solution $k$, and thus, the inter-temporal equilibrium starting from the initial stock $k_{0}=$ $K_{0} / N_{0}>0$ is unique.

We introduce a new assumption on the utility function $U(c, d, m)$ in order to prove the existence and uniqueness of the inter-temporal equilibrium: 
Assumption 3: $c$ and $m$ are gross substitutes to the good consumed during the second period of life $d$, that is $c_{R}^{\prime} \leq 0$ and $m_{R}^{\prime} \leq 0$.

A consequence of this assumption is that the savings function (8) increases with $R$. Indeed, as (6), (7) and (8) are such that (4) holds, we have:

$$
s_{R}^{\prime}=-c_{R}^{\prime}-\phi m_{R}^{\prime} \geq 0
$$

Example 1 : Let us assume that the utility function $U$ is additively separable, following $U(c, d, m)=u_{1}(c)+u_{2}(d)+v(m)$, with $u_{1}^{\prime}>0, u_{2}^{\prime}>0, v^{\prime}>0$, $u_{1}^{\prime \prime}<0, u_{2}^{\prime \prime}<0, v^{\prime \prime}<0$. In that case, assumption 3 is satisfied iif the elasticity of $u_{2}^{\prime}(d)$ is not smaller than $-1: d u_{2}^{\prime \prime}(d) / u_{2}^{\prime}(d) \geq-1$.

The following proposition proves the existence and uniqueness of the intertemporal equilibrium.

Proposition 2 : Under assumptions 1, 2 and 3, $\forall k_{0}>0$, a unique intertemporal equilibrium $\left(k_{t}\right)_{t \geq 0}$ exists starting from a given initial condition $k_{0}>$ 0 .

Proof: The proof establishes that for all $w>0$, a unique value $k>0$ exists, such that $\Delta(w, k)=0$. Let us first note that $\Delta$ is a strictly increasing function of $k$. Indeed,

$$
\Delta_{k}^{\prime}(w, k)=m\left(w, f^{\prime}(k)\right)+k m_{R}^{\prime} f^{\prime \prime}(k)-s_{R}^{\prime} f^{\prime \prime}(k) \geq m\left(w, f^{\prime}(k)\right)>0
$$

Thus, uniqueness is guaranteed.

The existence is proved by studying the limits of $\Delta$ for $k \rightarrow 0$ and $k \rightarrow \infty$. When $k$ goes to 0 , it can be bounded in such a way that: $k<1 \Rightarrow f^{\prime}(k)>$ $f^{\prime}(1)$. We then obtain the following inequalities:

$$
m\left(w, f^{\prime}(k)\right) \leq m\left(w, f^{\prime}(1)\right), s\left(w, f^{\prime}(k)\right) \geq s\left(w, f^{\prime}(1)\right)
$$

and thus:

$$
\Delta(w, k) \leq k m\left(w, f^{\prime}(1)\right)-s\left(w, f^{\prime}(1)\right)
$$

Finally we have:

$$
\lim _{k \rightarrow 0} \Delta(w, k) \leq-s\left(w, f^{\prime}(1)\right)<0
$$

When $k$ goes to $+\infty$, it can be bounded in such a way that: $k>1 \Rightarrow$ $f^{\prime}(k)<f^{\prime}(1)$. We then obtain the following inequality:

$$
\Delta(w, k) \geq k m\left(w, f^{\prime}(1)\right)-s\left(w, f^{\prime}(1)\right)
$$

Thus:

$$
\lim _{k \rightarrow+\infty} \Delta(w, k)=+\infty
$$

The continuity of $\Delta$ makes it possible to conclude that $k$ exists, such that $\Delta(w, k)=0$. 


\subsection{Converging inter-temporal equilibria}

Definition 2 An inter-temporal equilibrium $\left(K_{t}, N_{t}, c_{t}, d_{t}, m_{t}\right)_{t \geq 0}$ is said to be converging if the sequence $k_{t}=K_{t} / N_{t}$ converges to a limit $\bar{k}>0$ when $t$ goes to infinity.

If $k_{t}$ converges to a limit $\bar{k}$, it is straightforward to show that $R_{t}=R\left(k_{t}\right)$, $w_{t}=w\left(k_{t}\right), c_{t}, d_{t}$ and $m_{t}$ are converging to constant values $\bar{R}, \bar{w}, \bar{c}, \bar{d}$, and $\bar{m}$.

Definition 3 A converging inter-temporal equilibrium $\left(K_{t}, N_{t}, c_{t}, d_{t}, m_{t}\right)_{t \geq 0}$ is said to converge in under-accumulation if $\bar{R}>\bar{m}$. It is said to converge in over-accumulation if $\bar{R}<\bar{m}$.

An example of converging inter-temporal equilibria can be obtained when homothetic utility functions are considered. In this case, equations (6), (7), (8) and (9) take the following forms:

$$
\begin{aligned}
c_{t} & =w_{t} \gamma_{1}\left(R_{t+1}\right) \\
\phi m_{t} & =w_{t} \gamma_{3}\left(R_{t+1}\right) \\
s_{t} & =d_{t} / R_{t+1}=w_{t} \gamma_{2}\left(R_{t+1}\right)
\end{aligned}
$$

where the functions $\gamma_{i}(R)$ are such that: $\gamma_{1}(R)+\gamma_{2}(R)+\gamma_{3}(R)=1$. Assumption 3 is equivalent to $\gamma_{1}^{\prime}(R) \leq 0$ and $\gamma_{3}^{\prime}(R) \leq 0$, which implies $\gamma_{2}^{\prime}(R) \geq 0$.

The equilibrium dynamics (15) become:

$$
k_{t+1}=\frac{s_{t}}{m_{t}}=\frac{\phi \gamma_{2}\left(R_{t+1}\right)}{\gamma_{3}\left(R_{t+1}\right)}
$$

Under assumption 3, this equation has a unique solution $k_{t+1}=\bar{k}$. The economy reaches in one period its stationary state $\bar{k}$ defined by:

$$
\bar{k} \gamma_{3}[R(\bar{k})]=\phi \gamma_{2}[R(\bar{k})]
$$

This property comes from the homothetic utility function. As $s_{t}$ and $m_{t}$ are proportional to $w_{t}, k_{t+1}$ no longer depends on $w_{t}$. The two adjustments in savings and fertility lead to a constant ratio $K_{t+1} / N_{t+1}$ in one period.

\section{Efficient allocations and endogenous fertil- ity}

In this section, we show that the concept of Pareto optimality needs a new definition in a framework of endogenous fertility. We first introduce the basic 
notion of feasible allocations. This allows us to introduce different notions of efficiency. Finally, we give sufficient conditions for the non-efficiency of a competitive equilibrium.

\subsection{Feasible allocations with representative consumers}

First, we can define the notion of a feasible allocation with representative consumers as an inter-temporal allocation that satisfies the resource constraints of the economy.

Definition 4 (RC-allocation) A feasible allocation with representative consumers (or RC-allocation) is a sequence $\left(K_{t}, N_{t}, c_{t}, d_{t}, m_{t}\right)_{t \geq 0}$ of positive variables that satisfy $\forall t \geq 0$ :

$$
\begin{aligned}
& F\left(K_{t}, N_{t}\right)=K_{t+1}+N_{t} c_{t}+N_{t-1} d_{t}+N_{t+1} \phi \\
& N_{t+1}=m_{t} N_{t}
\end{aligned}
$$

For a competitive equilibrium, the initial conditions $N_{-1}, N_{0}, K_{0}$ and $d_{0}=$ $R_{0} K_{0} / N_{-1}$ are given. Following definition 4, an inter-temporal equilibrium is a feasible allocation, which satisfies equations (10), (11) and (14). From assumption 1 , if $K_{0}, N_{0}$ and $N_{-1}$ are positive, all the variables $\left(K_{t}, N_{t}, c_{t}\right.$, $\left.d_{t}, m_{t}\right)_{t \geq 0}$ will be positive.

Remark 1 To simplify the presentation, we will always consider the four initial quantities $\left(N_{-1}, N_{0}, K_{0}, d_{0}\right)$ as given. Thus, it will be hereafter implicit that all feasible allocations or competitive equilibria considered will start from these same initial conditions, even if they are not mentioned.

A new characterization of feasible RC-allocations will be useful in the sequel. In equation (22), $c_{t}$ and $m_{t}$ are chosen by generation $t$ agents when $d_{t}$ is determined by generation $t-1$ agents. By a simple change in variables, we can introduce a new definition of feasible allocations, in which all variables are decided by the same generation.

Lemma 1 (Characterizations of feasible RC-allocations) Any feasible $R C$-allocation can be characterized as a sequence $\left(Y_{t}, N_{t}, C_{t}, D_{t}, Z_{t}\right)_{t \geq 0}$ of positive variables starting from the initial condition $Y_{0}=F\left(K_{0}, N_{0}\right)-D_{0}$, and satisfying $\forall t \geq 0$,

$$
\begin{aligned}
& Y_{t+1}=F\left(Y_{t}-C_{t}-\phi Z_{t}, Z_{t}\right)-D_{t+1} \\
& N_{t+1}=Z_{t} \\
& Y_{t}>C_{t}+\phi Z_{t}
\end{aligned}
$$


Proof: This result is immediately obtained by a simple change in variables: $C_{t}=N_{t} c_{t}, D_{t}=N_{t-1} d_{t}$ and $Y_{t}=K_{t+1}+C_{t}+\phi Z_{t}$.

Remark 2 Let $\left(\bar{K}_{t}, \bar{N}_{t}, \bar{c}_{t}, \bar{d}_{t}, \bar{m}_{t}\right)_{t>0}$ be an inter-temporal equilibrium. Along such a trajectory, the new variable $Y_{t}$ is equal to:

$$
\bar{Y}_{t}=\bar{N}_{t} \bar{w}_{t}=\bar{N}_{t} F_{L}^{\prime}\left(\bar{K}_{t}, \bar{N}_{t}\right)
$$

We restrict our analysis to allocations that give the same levels of consumption and fertility for agents belonging to the same generation (RCallocations). As agents are identical within a generation, we will henceforth use the terms "the utility of a generation" to mean the utility level obtained by each agent belonging to this generation. In no way, this expression refers to an evaluation of the well-being of a generation measured by some social criterion. It is only a short cut to designate the utility level of each agent within a given generation.

\subsection{Efficiency of RC-allocations}

Pareto-efficiency is usually defined for a given set of agents. The model with endogenous fertility introduces a new difficulty, because the set of agents is the result of individual behaviors. Moreover, we only consider the case of identical agents within each generation, and we need a definition allowing to compare RC-feasible allocation.

In a basic framework with exogenous fertility and heterogeneous agents, the standard definition of Pareto-dominance would be the following. A first feasible allocation Pareto-dominates a second one if each agent in the first allocation obtains at least as much utility as in the second one, with a strict improvement for at least one agent. If we restrict the analysis to RC-allocations (allocations with identical agents within each generation), a first feasible allocation Pareto-dominates a second one if each generation in the first one obtains at least as much utility as in the second one, with a strict improvement for at least one generation ${ }^{5}$. As all agents within a generation are identical, the first allocation must not only improve the utility of one agent in one generation, but of all the agents of this generation too.

Finally, we have to deal with endogenous fertility. We introduce a first concept that is only concerned with the utility of each generation.

\footnotetext{
${ }^{5}$ By the utility of a generation, we again refer to the utility of each agent belonging to this generation.
} 
Definition 5 (RC-dominance) ${ }^{6}$ Let $\left(K_{t}^{i}, N_{t}^{i}, c_{t}^{i}, d_{t}^{i}, m_{t}^{i}\right)_{t \geq 0}$ for $i=1,2$ be two feasible $R C$-allocations. Allocation 1 is said to $R C$-dominates allocation 2 if it leads to a higher level of utility for all generations, with a strict improvement for (at least) one generation. Formally,

$$
\begin{aligned}
\forall t & \geq 0, U\left(c_{t}^{1}, d_{t+1}^{1}, m_{t}^{1}\right) \geq U\left(c_{t}^{2}, d_{t+1}^{2}, m_{t}^{2}\right) \\
\exists t_{0} & \geq 0, \text { such that: } U\left(c_{t_{0}}^{1}, d_{t_{0}+1}^{1}, m_{t_{0}}^{1}\right)>U\left(c_{t_{0}}^{2}, d_{t_{0}+1}^{2}, m_{t_{0}}^{2}\right)
\end{aligned}
$$

In the sense of this definition, the only criterion is the utility of each representative consumer within each generation. Thus, a feasible allocation that improves the utility level of one (or many) generation(s) to the detriment of the size of one (or many) generation(s) is considered preferable. This notion can be criticized, because it makes it possible to diminish the number of agents. For that reason, it is possible to think of a second notion, for which an allocation can dominate another only if it includes at least an equal number of children in each period. We call this alternative notion CRC-dominance ${ }^{7}$. Formally, we must add to the preceding definition the constraints:

$$
\forall t \geq 0, m_{t}^{1} \geq m_{t}^{2}
$$

It is straightforward that CRC-dominance implies RC-dominance, but the converse is a priori false.

From these notions of dominance, it follows two notions of efficiency:

Definition 6 An $R C$-allocation is said to be $R C$-efficient (respectively $C R C$ efficient), if there does not exist another $R C$-allocation that $R C$-dominates (respectively CRC-dominates) it.

It is straightforward that a RC-efficient allocation also is CRC-efficient, but the converse a priori is false. We will mainly concentrate on the first notion (RC-efficiency). Indeed, as a result of our study, it will appear that these two notions lead to the same classification of competitive equilibria. The reason behind this result is that non-RC-efficient equilibria are dominated by allocations that include a higher level of fertility.

\subsection{Conditions that ensure the non-RC-efficiency of a competitive equilibrium}

In this section, we prove two results. The first one shows that a competitive equilibrium that converges in over-accumulation is not RC-efficient (and not

\footnotetext{
${ }^{6} \mathrm{RC}$-dominance means dominance for Representative Consumers.

${ }^{7} \mathrm{CRC}$-dominance means dominance in Children for Representative Consumers.
} 
CRC-efficient). It is a direct extension of the standard result for an economy with exogenous fertility.

The second result is more surprising. It shows that a competitive equilibrium that converges in under-accumulation can be RC-dominated (and CRC-dominated). This result is shown using a simple example.

\subsubsection{The in-efficiency of an inter-temporal equilibrium that con- verges in over-accumulation $(\bar{m}>\bar{R})$}

We consider that $\left(\bar{K}_{t}, \bar{N}_{t}, \bar{c}_{t}, \bar{d}_{t}, \bar{m}_{t}\right)_{t \geq 0}$ is an inter-temporal equilibrium that converges in over-accumulation: $\bar{m}_{t}$ and $\bar{R}_{t}=F_{K}^{\prime}\left(\bar{K}_{t}, \bar{N}_{t}\right)$ respectively converge into $\bar{m}$ and $\bar{R}$, such that $\bar{m}>\bar{R}$. Thus, such an equilibrium is not RC-efficient. Indeed, we know that such a trajectory is not Pareto-optimal with an exogenous population, that is, when this allocation is compared to feasible allocations with the same population $\left(\forall t, m_{t}=\bar{m}_{t}\right)$. Thus, this allocation cannot be RC-efficient with endogenous fertility.

This first result gives a necessary condition for a converging equilibrium to be RC-efficient: it must converge in under-accumulation or towards the "golden rule" $(\bar{m}=\bar{R})$. When fertility is exogenous, converging in underaccumulation is a sufficient condition for an equilibrium to be Pareto-optimal. When fertility is endogenous, the question is to know if this result still holds.

\subsubsection{A competitive equilibrium that converges in under-accumulation can be non-RC-efficient}

This property is shown in an example. We use a log-linear utility function and a CES production function:

$$
\begin{aligned}
U_{t} & =\gamma_{1} \ln c_{t}+\gamma_{2} \ln d_{t+1}+\gamma_{3} \ln m_{t} \\
F(K, L) & =A\left(K^{\frac{\tau-1}{\tau}}+b L^{\frac{\tau-1}{\tau}}\right)^{\frac{\tau}{\tau-1}}
\end{aligned}
$$

The economy reaches the stationary state $\bar{k}=\phi \gamma_{2} / \gamma_{3}$ in one period. The following result is proved:

Proposition 3 For utility and production functions given by (24) and (25), if the following conditions hold,

$$
\begin{aligned}
\gamma_{3} & <\phi / b<\gamma_{2} \\
\phi / b-\gamma_{3} & <\frac{\left(\gamma_{2}-\gamma_{3}\right)^{2}}{4 \gamma_{2}}
\end{aligned}
$$


and if the elasticity of production $\tau$ is large enough, the stationary equilibrium $\bar{k}$ is in under-accumulation and a feasible allocation, which offers the same levels for consumption and a higher fertility level, exists. Thus, this allocation (C)RC-dominates the competitive equilibrium.

Proof: see appendix 1

Conditions (26) and (27) can be understood as follows. The inequality $\gamma_{3}<\phi / b$ ensures that the competitive equilibrium is associated with underaccumulation for $\tau$ when it is large enough. The inequality $\phi / b<\gamma_{2}$ makes it possible to define a feasible allocation that $(\mathrm{C}) \mathrm{RC}$-dominates the competitive equilibrium, such that the fertility level is higher than $\bar{R}$. Finally, (27) ensures that the competitive equilibrium $\bar{R}$ is close to $\bar{m}$.

The interpretation of the result is easier at the limit for $\tau=+\infty$. At each period, along the dominating trajectory, the economy accumulates less capital, because more resources are devoted to child-rearing. Thus, capital accumulation diminishes when the quantity of labor increases. The more the production factors are substitutable in the technology, the smaller the loss in production. When $\tau=+\infty$, the gross return on capital is constant and equal to $A$. Along the dominating trajectory, the fertility level $m$ is fixed at a value higher than $A=\bar{R}$.

\section{Sufficient conditions for the RC-efficiency of an inter-temporal equilibrium}

In this section, using general functions for utility and production, we give a sufficient condition that ensures that a converging equilibrium in underaccumulation is RC-efficient.

We consider feasible RC-allocations in the sense of the characterization in lemma 1. Thus, a feasible RC-allocation is defined as a sequence of aggregated variables $\left(Y_{t}, N_{t}, C_{t}, D_{t}, Z_{t}\right)_{t \geq 0}$.

The following lemma introduces a new function $V$ and gives its properties.

Lemma 2 The function $V(C, D, Z, N) \equiv N U\left(\frac{C}{N}, \frac{D}{N}, \frac{Z}{N}\right)$ defined on $\mathbb{R}_{++}^{4}$ is concave and twice continuously differentiable. Its partial derivatives are:

$$
V_{1}^{\prime}=U_{c}^{\prime}, \quad V_{2}^{\prime}=U_{d}^{\prime}, \quad V_{3}^{\prime}=U_{m}^{\prime} \quad \text { and } \quad V_{4}^{\prime}=U-\frac{C}{N} U_{c}^{\prime}-\frac{D}{N} U_{d}^{\prime}-\frac{Z}{N} U_{m}^{\prime}
$$

with $U$ and its partial derivatives taken at $\left(\frac{C}{N}, \frac{D}{N}, \frac{Z}{N}\right)$.

Proof: see appendix 2

We introduce a new lemma below, which gives a sufficient condition that ensures the RC-efficiency of an allocation. 
Lemma 3 Let $\left(\bar{Y}_{t}, \bar{N}_{t}, \bar{C}_{t}, \bar{D}_{t}, \bar{Z}_{t}\right)_{t \geq 0}$ be a feasible $R C$-allocation. Let $\left(\beta_{t}\right)$ be a sequence, such that $\forall t, \beta_{t}>0$. For a feasible $R C$-allocation $\left(Y_{t}, N_{t}, C_{t}\right.$, $\left.D_{t}, Z_{t}\right)_{t \geq 0}$, we define the sequence of gains:

$$
g_{t}\left(N_{t}, C_{t}, D_{t}, Z_{t}\right)=\beta_{t}\left[V\left(C_{t}, D_{t+1}, Z_{t}, N_{t}\right)-N_{t} U\left(\bar{C}_{t} / \bar{N}_{t}, \bar{D}_{t+1} / \bar{N}_{t}, \bar{Z}_{t} / \bar{N}_{t}\right)\right]
$$

If a sequence $\left(\beta_{t}\right)$ exists such that for all feasible RC-allocations $\left(Y_{t}, N_{t}, C_{t}\right.$, $\left.D_{t}, Z_{t}\right)_{t \geq 0}$

$$
\liminf _{T \rightarrow+\infty} \sum_{0}^{T}\left[g_{t}\left(N_{t}, C_{t}, D_{t}, Z_{t}\right)\right] \leq 0
$$

$\left(\bar{Y}_{t}, \bar{N}_{t}, \bar{C}_{t}, \bar{D}_{t}, \bar{Z}_{t}\right)_{t \geq 0}$ is RC-efficient.

Proof: Reductio ad absurdum. Let us assume that $\left(\bar{Y}_{t}, \bar{N}_{t}, \bar{C}_{t}, \bar{D}_{t}, \bar{Z}_{t}\right)_{t \geq 0}$ is not RC-efficient. In that case, it is RC-dominated by a feasible RC-allocation $\left(Y_{t}, N_{t}, C_{t}, D_{t}, Z_{t}\right)_{t \geq 0}$, such that:

$\forall t \geq 0, U\left(C_{t} / N_{t}, D_{t+1} / N_{t}, Z_{t} / N_{t}\right) \geq U\left(\bar{C}_{t} / \bar{N}_{t}, \bar{D}_{t+1} / \bar{N}_{t}, \bar{Z}_{t} / \bar{N}_{t}\right)$

$\exists t_{0} \geq 0$, such that $U\left(C_{t_{0}} / N_{t_{0}}, D_{t_{0}+1} / N_{t_{0}}, Z_{t_{0}} / N_{t_{0}}\right)>U\left(\bar{C}_{t_{0}} / \bar{N}_{t_{0}}, \bar{D}_{t_{0}+1} / \bar{N}_{t_{0}}, \bar{Z}_{t_{0}} / \bar{N}_{t_{0}}\right)$

For this allocation, when $T \geq t_{0}$

$$
\begin{aligned}
& \sum_{t=0}^{T} \beta_{t} N_{t}\left[U\left(C_{t} / N_{t}, D_{t+1} / N_{t}, Z_{t} / N_{t}\right)-U\left(\bar{C}_{t} / \bar{N}_{t}, \bar{D}_{t+1} / \bar{N}_{t}, \bar{Z}_{t} / \bar{N}_{t}\right)\right] \\
\geq & \beta_{t_{0}} N_{t_{0}}\left[U\left(C_{t_{0}} / N_{t_{0}}, D_{t_{0}+1} / N_{t_{0}}, Z_{t_{0}} / N_{t_{0}}\right)-U\left(\bar{C}_{t_{0}} / \bar{N}_{t_{0}}, \bar{D}_{t_{0}+1} / \bar{N}_{t_{0}}, \bar{Z}_{t_{0}} / \bar{N}_{t_{0}}\right)\right]>0
\end{aligned}
$$

and thus:

$$
\liminf _{T \rightarrow+\infty} \sum_{0}^{T}\left[g_{t}\left(N_{t}, C_{t}, D_{t}, Z_{t}\right)\right]>0
$$

which contradicts the assumption (29).

Remark 3 The assumption of the preceding lemma simply means that $\left(\bar{Y}_{t}\right.$, $\left.\bar{N}_{t}, \bar{C}_{t}, \bar{D}_{t}, \bar{Z}_{t}\right)_{t \geq 0}$ is weakly maximal in the sense of Brock [3]. Indeed, as $g_{t}\left(\bar{N}_{t}, \bar{C}_{t}, \bar{D}_{t}, \bar{Z}_{t}\right)=0$, (29) can also be written as:

$$
\liminf _{T \rightarrow+\infty} \sum_{0}^{T}\left[g_{t}\left(N_{t}, C_{t}, D_{t}, Z_{t}\right)-g_{t}\left(\bar{N}_{t}, \bar{C}_{t}, \bar{D}_{t}, \bar{Z}_{t}\right)\right] \leq 0
$$

No feasible allocation overtakes $\left(\bar{Y}_{t}, \bar{N}_{t}, \bar{C}_{t}, \bar{D}_{t}, \bar{Z}_{t}\right)_{t \geq 0}$. 
The use of aggregate variables in the definition of feasible RC-allocations $\left(Y_{t}, N_{t}, C_{t}, D_{t}, Z_{t}\right)_{t \geq 0}$ is justified for the following reason. By lemma 2, we know that $V$ is concave. Thus, the function of gain $g_{t}$ is concave. With the condition (23), the set of feasible allocations is convex. Thus, the optimization problem defined by the gain function (28) in the set of feasible allocations is concave.

The following proposition gives sufficient conditions for a competitive equilibrium converging in under-accumulation to be RC-efficient.

Proposition 4 Let $\left(\bar{K}_{t}, \bar{N}_{t}, \bar{c}_{t}, \bar{d}_{t}, \bar{m}_{t}\right)_{t \geq 0}$ be a competitive equilibrium that is converging towards a steady state in under-accumulation: $\bar{R}>\bar{m}$. If $\phi \bar{R}>\bar{w}$, this equilibrium is $R C$-efficient.

Proof: see appendix 3.

The proof uses lemma 3 with an appropriate choice of the sequence $\left(\beta_{t}\right)$. First, we prove that the competitive equilibrium satisfies the first order conditions of the optimization program. The gain function has been defined by (28) in such a way that makes it possible. Second, we show that the transversality condition is satisfied if $\phi \bar{R}>\bar{w}$. This result is consistent with the intuition from Section 4.3.2: to RC-dominate a competitive equilibrium converging in under-accumulation, a feasible RC-allocation must lead to a fertility level that is higher than $\bar{R}$. The constraint $\phi \bar{R}>\bar{w}$ rules out this possibility. Indeed, this inequality implies that, even if all the revenue is spent on children, the resulting fertility level $m=\bar{w} / \phi$ will be smaller than $\bar{R}$.

Remark 4 The condition $\phi \bar{R}>\bar{w}$ is the same as the assumption made in Becker and Barro [2]. In their framework, they need this condition to obtain an equilibrium with operative bequests.

It is important to note that the condition $\phi \bar{R}>\bar{w}$ is stronger than underaccumulation. Indeed, at the steady state of the competitive economy, we have: $\phi \bar{m} \leq \bar{w}<\phi \bar{R} \Rightarrow \bar{m}<\bar{R}$.

Remark 5 In an economy endowed with CES production and utility functions, Michel and Wigniolle [11] proves a stronger result. Let $\tau$ and $\rho$ respectively be the elasticities of substitution for production and utility functions. Assume that $\tau+\rho \geq 1$ (the competitive equilibrium is determined), that $\tau \leq 1$ (production factors are complements in production) and that the competitive economy converges in under-accumulation $(\bar{R}>\bar{m})$. Then, if $\tau+\rho \leq 2$, the competitive equilibrium is $R C$-efficient. In this case, underaccumulation implies RC-efficiency. 


\section{Conclusion}

This paper has studied the efficiency of the competitive equilibrium for an overlapping generations economy with endogenous fertility. We have discussed the notion of efficiency that fit in with endogenous fertility: RCefficiency or CRC-efficiency. We have proved that a competitive equilibrium that converges in over-accumulation is non-RC-efficient. Using an example, however, we have shown that under-accumulation is not a sufficient condition for ensuring RC-efficiency: a competitive equilibrium that converges in under-accumulation can be non-RC-efficient. Finally, we have proved a sufficient condition ensuring the RC-efficiency of the competitive equilibrium.

\section{Appendix 1: proof of proposition 3.}

With a log linear utility function given by (24), we can use equations (17), (18), (19) and (21) with constant values for $\gamma_{i}, i=1,2,3$. We assume that the competitive equilibrium is in under-accumulation. This property is expressed by the condition:

$$
\bar{R}>\bar{m}=\frac{\gamma_{3} \bar{w}}{\phi} \Leftrightarrow \gamma_{3}<\frac{\phi \bar{R}}{\bar{w}}
$$

We can then build a feasible RC-allocation starting from the stationary state $k_{0}=\bar{k}$ and $m_{-1}=\bar{m}$, such that: $\forall t \geq 1, c_{t}=\bar{c}, d_{t}=\bar{d}, m_{t}=m$, and $k_{t}$ satisfies the resource constraint:

$$
m k_{t+1}=f\left(k_{t}\right)-\bar{c}-\frac{\bar{d}}{m}-\phi m
$$

In $t=0$, as $m_{-1}$ is given, the resource constraint is:

$$
m k_{1}=f(\bar{k})-\bar{c}-\frac{\bar{d}}{\bar{m}}-\phi m
$$

As $\bar{d}=\bar{R} \bar{s}=\bar{R} \bar{m} \bar{k}$, the term $f(\bar{k})-\bar{d} / \bar{m}$ is equal to $f(\bar{k})-\overline{R k}=\bar{w}$. Thus, the precedent condition becomes:

$$
m k_{1}=\bar{w}-\gamma_{1} \bar{w}-\phi m
$$

We choose $m=\mu$, with $\mu$ defined as $\mu^{2} \equiv \bar{d} / \phi=\gamma_{2} \bar{R} \bar{w} / \phi \cdot m=\mu$ is the value that minimizes the term $d / m+\phi m$ in equation (31). At each date $t$, the condition $k_{t}>0$ must hold. As

$$
f(k)>w(k)=A b\left(k^{\frac{\tau-1}{\tau}}+b\right)^{\frac{1}{\tau-1}}>A b^{\frac{\tau}{\tau-1}}
$$


we obtain from (32) and (31) sufficient conditions that respectively ensure that $k_{1}>0$ and $k_{t+1}>0 \forall t \geq 1$ :

$$
\begin{aligned}
A b^{\frac{\tau}{\tau-1}}-\gamma_{1} \bar{w}-\phi \mu & >0 \\
A b^{\frac{\tau}{\tau-1}}-\gamma_{1} \bar{w}-2 \phi \mu & >0
\end{aligned}
$$

Thus, the only condition that must hold is the last one (33).

Finally, we impose the inequality $\mu>\bar{R}$, which leads to:

$$
\phi \bar{R} / \bar{w}<\gamma_{2}
$$

Conditions (30) and (34) imply: $\mu>\bar{R}>\bar{m}$ and then $U(\bar{c}, \bar{d}, \mu)>$ $U(\bar{c}, \bar{d}, \bar{m})$. All utilities are higher along the feasible trajectory with $m_{t}=\mu$.

The three conditions (30), (33) and (34) remain to be studied at the limit when $\tau \rightarrow+\infty$. When $\tau \rightarrow+\infty, \lim \bar{R}=A$ and $\lim \bar{w}=A b$.

We assume that: $\gamma_{3}<\gamma_{2}$. Conditions (30) and (34) become:

$$
\gamma_{3}<\phi / b<\gamma_{2}
$$

Condition (33) can be written as:

$$
2 \phi \mu<A b\left(1-\gamma_{1}\right)=A b\left(\gamma_{2}+\gamma_{3}\right)
$$

Taking the square of the precedent inequality, we obtain:

$$
4 \phi^{2} \mu^{2}=4 \phi \gamma_{2} A^{2} b<A^{2} b^{2}\left(\gamma_{2}+\gamma_{3}\right)^{2}
$$

In setting $\varepsilon=\phi / b-\gamma_{3}$ and replacing $\phi / b$ by $\varepsilon+\gamma_{3}$, we have:

$$
\varepsilon<\frac{\left(\gamma_{2}-\gamma_{3}\right)^{2}}{4 \gamma_{2}}
$$

Thus, when conditions (26) and (27) in the text are satisfied, conditions (30), (33) and (34) hold in the limit case when $\tau \rightarrow+\infty$. By continuity, they are satisfied when $\tau$ is large enough.

\section{Appendix 2: proof of lemma 2}

We can introduce the following notation: $X=(C, D, Z, N)$. For $\lambda \in$ $(0,1)$, we note $\bar{X}=\lambda X_{1}+(1-\lambda) X_{2}, \bar{C}=\lambda C_{1}+(1-\lambda) C_{2}$, etc.. Thus, we can write:

$$
\begin{gathered}
V(\bar{X})=\bar{N} U\left(\frac{\bar{C}}{\bar{N}}, \frac{\bar{D}}{\bar{N}}, \frac{\bar{Z}}{\bar{N}}\right)= \\
=\bar{N} U\left(\lambda \frac{N_{1}}{\bar{N}} \frac{C_{1}}{N_{1}}+(1-\lambda) \frac{N_{2}}{\bar{N}} \frac{C_{2}}{N_{2}}, \lambda \frac{N_{1}}{\bar{N}} \frac{D_{1}}{N_{1}}+(1-\lambda) \frac{N_{2}}{\bar{N}} \frac{D_{2}}{N_{2}}, \lambda \frac{N_{1}}{\bar{N}} \frac{Z_{1}}{N_{1}}+(1-\lambda) \frac{N_{2}}{\bar{N}} \frac{Z_{2}}{N_{2}}\right)
\end{gathered}
$$


As $\lambda \frac{N_{1}}{\bar{N}}+(1-\lambda) \frac{N_{2}}{\bar{N}}=1$ and $U$ concave, we have:

$$
V(\bar{X}) \geq \bar{N} \lambda \frac{N_{1}}{\bar{N}} U\left(\frac{C_{1}}{N_{1}}, \frac{D_{1}}{N_{1}}, \frac{Z_{1}}{N_{1}}\right)+\bar{N}(1-\lambda) \frac{N_{2}}{\bar{N}} U\left(\frac{C_{2}}{N_{2}}, \frac{D_{2}}{N_{2}}, \frac{Z_{2}}{N_{2}}\right)
$$

or finally:

$$
V(\bar{X}) \geq \lambda V\left(X_{1}\right)+(1-\lambda) V\left(X_{2}\right)
$$

Thus, $V$ is concave.

$V$ is twice continuously differentiable as $U$. The partial derivatives are straightforward.

\section{Appendix 3: proof of proposition 4}

We prove proposition 4 in three steps. First, we make explicit a class of optimization programs which solutions give RC-efficient allocations. Second, we prove that a competitive equilibrium satisfies the first order conditions of such an optimization program. Third we prove that under the assumption given in proposition 4 , the transversality condition also holds along the competitive equilibrium trajectory.

\section{The optimization program}

The proof uses lemma 3 with an optimization program associated with the gain function:

$$
g_{t}\left(N_{t}, C_{t}, D_{t}, Z_{t}\right)=\beta_{t}\left[V\left(C_{t}, D_{t+1}, Z_{t}, N_{t}\right)-N_{t} U\left(\bar{C}_{t} / \bar{N}_{t}, \bar{D}_{t+1} / \bar{N}_{t}, \bar{Z}_{t} / \bar{N}_{t}\right)\right]
$$

for an appropriate choice of the sequence $\left(\beta_{t}\right)$, and under the two dynamical constraints:

$$
\begin{aligned}
& Y_{t+1}=F\left(Y_{t}-C_{t}-\phi Z_{t}, Z_{t}\right)-D_{t+1} \\
& N_{t+1}=Z_{t}
\end{aligned}
$$

From Michel [10], the conditions for weak maximality of this problem are obtained in using the Lagrangian:

$$
\begin{aligned}
\mathcal{L}_{t}= & \beta_{t}\left[V\left(C_{t}, D_{t+1}, Z_{t}, N_{t}\right)-V\left(\bar{C}_{t}, \bar{D}_{t+1}, \bar{Z}_{t}, \bar{N}_{t}\right)\right]+ \\
& \lambda_{t+1}\left[F\left(Y_{t}-C_{t}-\phi Z_{t}, Z_{t}\right)-D_{t+1}\right]-\lambda_{t} Y_{t}+\mu_{t+1} Z_{t}-\mu_{t} N_{t}
\end{aligned}
$$

with $\lambda_{t}$ and $\mu_{t}$ being the shadow prices associated with $Y_{t}$ and $N_{t}$.

We will use the abbreviated notations in the sequel:

$$
\begin{aligned}
U_{t} & =U\left(\frac{C_{t}}{N_{t}}, \frac{D_{t+1}}{N_{t}}, \frac{N_{t+1}}{N_{t}}\right)=U\left(c_{t}, d_{t+1}, m_{t}\right) \\
\bar{U}_{t} & =U\left(\bar{c}_{t}, \bar{d}_{t+1}, \bar{m}_{t}\right) \\
U_{\theta, t}^{\prime} & =U_{\theta}^{\prime}\left(\frac{C_{t}}{N_{t}}, \frac{D_{t+1}}{N_{t}}, \frac{N_{t+1}}{N_{t}}\right)=U_{\theta}^{\prime}\left(c_{t}, d_{t+1}, m_{t}\right) \text { with } \theta=c, d \text { or } m \\
F_{K_{t+1}}^{\prime} & =F_{K}^{\prime}\left(Y_{t}-C_{t}-\phi Z_{t}, Z_{t}\right) \\
F_{L_{t+1}}^{\prime} & =F_{L}^{\prime}\left(Y_{t}-C_{t}-\phi Z_{t}, Z_{t}\right)
\end{aligned}
$$


The first-order conditions are:

$$
\begin{aligned}
\beta_{t} U_{c, t}^{\prime} & =\lambda_{t+1} F_{K_{t+1}}^{\prime} \\
\beta_{t} U_{d, t}^{\prime} & =\lambda_{t+1} \\
\beta_{t} U_{m, t}^{\prime} & =\lambda_{t+1}\left(\phi F_{K_{t+1}}^{\prime}-F_{L_{t+1}}^{\prime}\right)-\mu_{t+1} \\
\mu_{t} & =\beta_{t}\left[U_{t}-\bar{U}_{t}-\frac{C_{t}}{N_{t}} U_{c, t}^{\prime}-\frac{D_{t+1}}{N_{t}} U_{d, t}^{\prime}-\frac{N_{t+1}}{N_{t}} U_{m, t}^{\prime}\right] \\
\lambda_{t} & =\lambda_{t+1} F_{K_{t+1}}^{\prime}
\end{aligned}
$$

The transversality condition is (cf. Michel [10]):

$$
\limsup _{t \rightarrow+\infty}\left(\lambda_{t} \widetilde{Y}_{t}+\mu_{t} \widetilde{N}_{t}-\lambda_{t} Y_{t}-\mu_{t} N_{t}\right) \geq 0
$$

for all feasible RC-allocations $\left(\widetilde{Y}_{t}, \widetilde{N}_{t}, \widetilde{C}_{t}, \widetilde{D}_{t}, \widetilde{Z}_{t}\right)_{t \geq 0}$ leading to a non-infinite loss with respect to the trajectory $\left(Y_{t}, N_{t}, C_{t}, D_{t}, Z_{t}\right)_{t \geq 0}$, that is that:

$$
\liminf _{T \rightarrow+\infty} \sum_{0}^{T}\left[g_{t}\left(\widetilde{Y}_{t}, \widetilde{N}_{t}, \widetilde{C}_{t}, \widetilde{D}_{t}, \widetilde{Z}_{t}\right)-g_{t}\left(Y_{t}, N_{t}, C_{t}, D_{t}, Z_{t}\right)\right]>-\infty
$$

As the optimization program is concave, the first order conditions and the transversality condition are necessary and sufficient for weak maximality (in the sense of Brock [3]). For an appropriate choice of the sequence $\left(\beta_{t}\right)$, if these maximality conditions are satisfied by the equilibrium trajectory $\left(\bar{Y}_{t}, \bar{N}_{t}, \bar{C}_{t}, \bar{D}_{t}, \bar{Z}_{t}\right)_{t \geq 0}$, from lemma 3 , this equilibrium is RC-efficient.

\section{The equilibrium trajectory satisfies the first order conditions}

We first prove that all the first order conditions are satisfied by the equilibrium trajectory $\left(\bar{Y}_{t}, \bar{N}_{t}, \bar{C}_{t}, \bar{D}_{t}, \bar{Z}_{t}\right)_{t \geq 0}$, when the sequence $\left(\beta_{t}\right)$ is defined by:

$$
\begin{aligned}
& \beta_{0}=1 \\
& \forall t \geq 1, \frac{\beta_{t}}{\beta_{t-1}}=\frac{U_{c}^{\prime}\left(\bar{c}_{t-1}, \bar{d}_{t}, \bar{m}_{t-1}\right)}{U_{c}^{\prime}\left(\bar{c}_{t}, \bar{d}_{t+1}, \bar{m}_{t}\right) F_{K}^{\prime}\left(\bar{K}_{t}, \bar{N}_{t}\right)}
\end{aligned}
$$

and for shadow prices, such that:

$$
\begin{aligned}
& \lambda_{t}=\beta_{t} \bar{U}_{c, t}^{\prime} \\
& \mu_{t}=-\beta_{t} \bar{U}_{c, t}^{\prime} F_{L}^{\prime}\left(\bar{K}_{t}, \bar{N}_{t}\right)=-\beta_{t} \bar{U}_{c, t}^{\prime} \bar{w}_{t}
\end{aligned}
$$

Equation (39) is satisfied from (41) and the definition of $\beta_{t}$. Using (39), (35) is satisfied from (41). (36) is satisfied, because along the competitive 
equilibrium, the maximization condition (11) is true: $\bar{U}_{c, t}^{\prime} / \bar{U}_{d, t}^{\prime}=F_{K}^{\prime}\left(\bar{K}_{t+1}, \bar{N}_{t+1}\right)$. (38) is equivalent to (42), as, along the competitive equilibrium:

$$
\frac{\bar{C}_{t}}{\bar{N}_{t}} \bar{U}_{c, t}^{\prime}+\frac{\bar{D}_{t+1}}{\bar{N}_{t}} \bar{U}_{d, t}^{\prime}+\frac{\bar{N}_{t+1}}{\bar{N}_{t}} \bar{U}_{m, t}^{\prime}=\bar{U}_{c, t}^{\prime}\left(\bar{c}_{t}+\frac{\bar{d}_{t+1}}{\bar{R}_{t+1}}+\phi \bar{m}_{t}\right)=\bar{U}_{c, t}^{\prime} \bar{w}_{t}
$$

Finally, (37) can be written:

$$
\mu_{t+1}=\beta_{t} \bar{U}_{c, t}^{\prime}\left(-\frac{\bar{U}_{m, t}^{\prime}}{\bar{U}_{c, t}^{\prime}}+\phi-\frac{\bar{w}_{t+1}}{\bar{R}_{t+1}}\right)=-\beta_{t} \bar{U}_{c, t}^{\prime} \frac{\bar{w}_{t+1}}{\bar{R}_{t+1}}
$$

as $\phi-\bar{U}_{m, t}^{\prime} / \bar{U}_{c, t}^{\prime}=0$ along a competitive equilibrium. Using (42) in $t+1$, we finally find:

$$
-\beta_{t+1} \bar{U}_{c, t+1}^{\prime} \bar{w}_{t+1}=-\beta_{t} \bar{U}_{c, t}^{\prime} \frac{\bar{w}_{t+1}}{\bar{R}_{t+1}}
$$

which is true from the definition of the sequence $\left(\beta_{t}\right)$.

Thus, along the equilibrium trajectory, all the first order conditions of the optimization problem are satisfied. The transversality condition is the only condition that remains to be proved.

As $\lambda_{t} \bar{Y}_{t}+\mu_{t} \bar{N}_{t}=\beta_{t} \bar{U}_{c, t}^{\prime} \bar{N}_{t}\left(\bar{w}_{t}-\bar{w}_{t}\right)=0,(40)$ can be written as:

$$
\limsup _{t \rightarrow+\infty} \beta_{t} \bar{U}_{c, t}^{\prime}\left(\widetilde{Y}_{t}-\bar{w}_{t} \widetilde{N}_{t}\right) \geq 0
$$

or:

$$
\limsup _{t \rightarrow+\infty} \beta_{t} \bar{U}_{c, t}^{\prime} \widetilde{N}_{t}\left(\widetilde{m}_{t} \widetilde{k}_{t+1}+\widetilde{c}_{t}+\phi \widetilde{m}_{t}-\bar{w}_{t}\right) \geq 0
$$

\section{The equilibrium trajectory satisfies the transversality condition}

Two cases may occur.

If $\limsup \widetilde{m}_{t} \widetilde{k}_{t+1}+\widetilde{c}_{t}+\phi \widetilde{m}_{t}-\bar{w}_{t}>0$, as $\beta_{t} \bar{U}_{c, t}^{\prime} \widetilde{N}_{t}>0$, the transversality condition is satisfied.

If $\limsup _{t \rightarrow+\infty} \widetilde{m}_{t} \widetilde{k}_{t+1}+\widetilde{c}_{t}+\phi \widetilde{m}_{t}-\bar{w}_{t} \leq 0$, the transversality condition is not necessarily satisfied. However, if $\bar{w}<\phi \bar{R}, \varepsilon>0$ exists, such that for $t$ large enough, $\phi \widetilde{m}_{t}<\bar{w}_{t}<\phi(\bar{R}-\varepsilon)$. Thus, $t$ being large enough, $\widetilde{m}_{t}<\bar{R}-\varepsilon$, and a constant $B$ exists, such that $\widetilde{N}_{t}<B(\bar{R}-\varepsilon)^{t}$. As

$$
\frac{\beta_{t} \bar{U}_{c, t}^{\prime}}{\beta_{t-1} \bar{U}_{c, t-1}^{\prime}}=\frac{1}{\bar{R}_{t}} \rightarrow \frac{1}{\bar{R}}
$$

we finally have:

$$
\lim _{t \rightarrow+\infty} \beta_{t} \bar{U}_{c, t}^{\prime} \widetilde{N}_{t}=0
$$


Thus, the transversality condition is satisfied. We have proved that a competitive equilibrium that converges in under-accumulation towards a stationary state, such that $\bar{w}<\phi \bar{R}$ is RC-efficient.

\section{References}

1. Becker, G.S.: An economic analysis of fertility. In: Demographic and economic change in developed countries. Princeton, NJ: Princeton Univiversity Press 1960

2. Becker, G.S., Barro, R.J.: A reformulation of the economic theory of fertility. Quarterly Journal of Economics 103, 1-25 (1988)

3. Brock, W.A.: On the existence of weakly-maximal programmes in a multi-sector economy. Review of Economic Studies 37, 275-280 (1970)

4. Chakrabarti, R.: Endogenous fertility and growth in a model with old age support. Economic Theory 13, 393-416 (1999)

5. Conde-Ruiz, J.I., Gimenez, E.L., Perez-Nievas, M.: Efficiency in an overlapping generations model with endogenous population, mimeo (2002)

6. De la Croix, D., Michel, P.: A theory of economic growth: dynamics and policy in overlapping generations. Cambridge: Cambridge University Press 2002

7. Diamond, P.: National debt in a neoclassical growth model. American Economic Review 55, 1126-1150 (1965)

8. Eckstein, Z., Wolpin, K.: Endogenous fertility and optimal population size. Journal of Public Economics 27, 93-106 (1985)

9. Golosov, M., Jones, L.E., Tertilt, M.: Efficiency with endogenous population growth. NBER 10231 (2004)

10. Michel, P.: Some clarifications on the transversality conditions. Econometrica 58, 705-723 (1990)

11. Michel, P., Wigniolle B.: On efficient child making. cahier de la MSE 2003.94, (2003)

12. Nishimura, K., Zhang, J.: Pay-as-you-go public pensions with endogenous fertility. Journal of Public Economics 48, 239-258 (1992) 
13. Raut, L.K., Srinivasan, T.N.: Dynamics of endogenous growth. Economic Theory 4, 777-90 (1994)

14. Willis, R.: A new approach to the economic theory of fertility behavior. Journal of Political Economy 81, S14-S64 (1973) 